\title{
SERVIÇOS DE SAÚDE E COMUNIDADES TERAPÊUTICAS: HÁ UMA RELAÇÃO EM PROL DO DEPENDENTE QUÍMICO?
}

\section{Iuri Fernando Coutinho e Silva Kévin da Silva Souza}

Graduando em Medicina pela Pontifícia Universidade Católica de Goiás (PUC Goiás), Brasil;

E-mail: iuri.fernando@hotmail.com

\section{Sonis Henrique Rezende Batista}

Mestrando em Ciências Ambientais e Saúde pela Pontifícia Universidade Católica de Goiás (PUC Goiás), Brasil.

Rogério José de Almeida

Pós-Doutorando em Ciências da Saúde pela Universidade Federal de Goiás (UFG); Docente do Departamento de Medicina e do Programa de Pós-Graduação em Ciências Ambientais e Saúde da Pontifícia Universidade Católica de Goiás (PUC Goiás), Brasil.
RESUMO: O uso de droga é um dos principais problemas de saúde pública em todo o mundo. Para o auxílio no tratamento aos dependentes químicos existem no Brasil alguns serviços, dentre eles se encontram as comunidades terapêuticas que são instituições não governamentais que devem funcionar de forma articulada com a atenção básica de saúde e com os Centros de Atenção Psicossocial (CAPS). O objetivo deste estudo foi analisar o relacionamento entre as comunidades terapêuticas e os serviços de atenção à saúde disponibilizados pelo Sistema Único de Saúde (SUS). Foram pesquisadas 43 comunidades localizadas no município de Goiânia (GO), sua região metropolitana e na cidade de Anápolis (GO). O trabalho de campo foi realizado por meio da aplicação de questionário. Todas as comunidades visitadas faziam uso do Sistema Único de Saúde e, na contramão desse constante uso, identificou-se que não há uma relação efetiva entre as comunidades e os CAPS. As comunidades possuem o direito e o dever de proporcionar aos residentes o acesso ao SUS garantido por lei. Já o CAPS, como instituição de serviço voltado para o tratamento e reinserção social dos usuários de drogas, serviria como um pilar, juntamente com as comunidades no auxílio ao dependente químico. A relação entre os serviços de saúde e as comunidades se apresenta, em princípio, em benefício dos dependentes químicos. No entanto, há a necessidade de haver uma maior interlocução das comunidades com os CAPS visando a reabilitação integral dos residentes.

PALAVRAS-CHAVE: Comunidade Terapêutica; Dependência Química; Saúde Pública.

\section{HEALTH SERVICES AND THERAPEUTIC COMMUNITIES: IS THERE A RELATIONSHIP FOR THE DRUG- DEPENDENT PATIENT?}

\begin{abstract}
One of the main problems in public health is the use of illicit drugs. Several services are an aid in the treatment of illicit drugdependent people among which may be mentioned the therapeutic communities. They are non-governmental organizations which function together with the Basic Health Care and with Centers for Psychosocial Care (SAPS). Current analysis deals with the relationship between therapeutic communities and Health Services available at the National Health Service (SUS). Forty-three communities were researched in the municipality of Goiânia GO Brazil, the metropolitan region and the city of Anápolis GO Brazil. Field work was undertaken with a questionnaire. All communities visited use SUS services, but there is no relationship between the communities and CAPS. Communities have the right and the duty to provide resident people access to SUS, which is warranted by law. As a service institution for the treatment and social reinsertion of illicit drug users, CAPS is an important institution for communities
\end{abstract}


in their aid for the drug user. The relationship between health service and communities is primarily for drug dependent people. However, greater interlocution must exist between the communities and CAPS for the integral rehabilitation of residents.

KEYWORDS: Therapeutic Community; DrugDependent; Public Health.

\section{INTRODUÇÃO}

O uso abusivo de drogas é um dos principais problemas de saúde pública em todo o mundo. O Relatório Mundial sobre Drogas do ano de 2012 (UNODC, 2012) mostrou que 3,4\% e 6,6\% da população mundial entre 15 e 64 anos é atingida pelo uso de drogas ilícitas. Já o Relatório Mundial de Drogas de 2015 apresentou que 1 entre 20 pessoas com a idade de 15 a 64 anos fez uso de alguma droga ilícita em 2013, sendo que 1 a cada 10 usuários de drogas tem algum problema relacionado ao uso, como transtornos ou dependência.

Observa-se também um aumento de doenças como HIV, hepatites B e C entre os usuários de drogas. O Relatório Mundial sobre Drogas de 2013 (UNODC, 2013) apresenta que das cerca de 14 milhões de pessoas que usavam drogas injetáveis no mundo, 1,6 milhões são portadoras de HIV, próximo de 7,2 milhões são portadoras do vírus da hepatite $C$ e 1,8 milhões são portadoras de hepatite B. O uso dessas substâncias ilícitas também interfere no número de mortes de um país, como apresenta uma pesquisa feita por Dias, Araújo e Laranjeira (2011), entre os anos de 1992 a 2006, em que mostrou que dos 131 dependentes estudados, 27 faleceram em decorrência do uso de drogas.

De acordo com o Relatório Mundial sobre Drogas de 2013, o uso de drogas é a causa principal de morte prematura entre a faixa etária de 26 a 44 anos. $\mathrm{O}$ uso e abuso de drogas deve ser entendido como um problema multidimensional e global, já que ele é fruto de um contexto socioeconômico, político e cultural (FERREIRA; LUIS VILLAR, 2004). O Brasil é um dos países onde há uma rota importante do tráfico de drogas, bem como um dos países que mais tem aumentado o consumo de drogas, principalmente a cocaína e o crack (DAMAS, 2013).
As ações estratégicas do governo brasileiro para reduzir o uso de drogas estão voltadas, sobretudo para ações de segurança pública, por meio do enfrentamento ao tráfico de drogas e da redução de oferta, de ações em saúde e assistência social na recuperação dos usuários e em educação e informação dirigida na prevenção do uso de drogas (DAMAS, 2013).

Há hoje no Brasil uma rede de serviços que ainda necessita de uma melhor interlocução entre os serviços visando à assistência aos dependentes. Pode-se citar, por exemplo, os serviços vinculados ao Sistema Único de Saúde (Centro de Atenção Psicossocial, Núcleo de Apoio à Saúde da Família, Estratégia de Saúde da Família e hospitais), aqueles que estão vinculados à assistência social (Centro de Referência de Assistência Social, Centro de Referência Especializado de Assistência Social), instituições religiosas e filantrópicas (Alcoólicos Anônimos, Narcóticos Anônimos, dentre outros), órgãos do Poder Judiciário (Delegacias, Ministério Público, Juizados), serviços privados (clínicas e ambulatórios especializados), e também as chamadas comunidades terapêuticas - CTs (BRASIL, 2011a).

Em se tratando das comunidades terapêuticas, estas devem funcionar como instituições sociais que buscam remediar, restaurar ou curar o dependente químico, procurando mudar de forma integral o indivíduo (DE LEON, 2003). Visam promover a reabilitação física e psicológica, recuperação, resgate da cidadania e reinserção social do indivíduo dependente por meio de um programa terapêutico que pode envolver laborterapia, convivência comunitária, desenvolvimento de atividades profissionalizantes, programas culturais, atividades religiosas e espirituais, atendimento às famílias, etc. (RAUPP; MILNITISKY-SAPIRO, 2008).

As comunidades terapêuticas estão enquadradas na Portaria $n^{0}$ 3088/2011 que prevê a criação, ampliação e articulação de serviços de cuidado à saúde para pessoas com sofrimento ou transtorno mental decorrentes do uso de drogas. Os dependentes químicos que se internam nestas instituições podem permanecer por até nove meses. Elas devem funcionar de forma articulada com a atenção básica (oferece os cuidados clínicos gerais ao dependente), com o Centro de Atenção Psicossocial - CAPS responsável pela indicação do acolhimento, 
acompanhamento durante o período que o indivíduo permanecer na comunidade e pelo planejamento de saída (BRASIL, 2011a).

Atualmente no Brasil, as comunidades devem seguir as determinações sanitárias da RDC 29/2011. De acordo com essa resolução, essas instituições devem ser diferenciadas dos equipamentos de saúde, constituindo-se como "equipamentos sociais". Nesse papel, as comunidades terapêuticas devem prestar, em regime de residência, serviços de atenção às pessoas com transtornos decorrentes do abuso de drogas, enquanto os equipamentos de saúde englobam hospitais, clínicas e congêneres (BRASIL, 2011b).

A RDC 29/2011 visa também a aproximação entre as comunidades e os serviços de saúde disponibilizados pelo SUS, já que ela define que a apresentação dos serviços de saúde aos atendidos por essas entidades fica a cargo da rede pública ou privada, a partir do encaminhamento à rede de atenção à saúde. Dessa forma, a instituição passa de equipamento social a serviço de saúde, caso os residentes desse estabelecimento necessitem de atendimento (BRASIL, 2011b).

Nesse sentido, considerando a necessária interlocução das comunidades terapêuticas com os serviços de saúde, tem-se como objetivo desta pesquisa analisar o atual relacionamento entre as comunidades terapêuticas com os serviços de saúde do SUS, principalmente no que se refere à atenção à saúde fora da comunidade para o atendimento aos residentes e as interlocuções com os Centros de Atenção Psicossocial CAPS.

\section{METODOLOGIA}

Foram pesquisadas um total de 43 comunidades terapêuticas, por intermédio de seus responsáveis, localizadas no município de Goiânia (GO) e região metropolitana, bem como a cidade de Anápolis (GO), utilizando-se de um roteiro semiestruturado, adaptado de Morselli, Rezende e Rocha (2012) que contêm: dados gerais dos entrevistados, dados gerais de identificação da comunidade, serviços oferecidos pela comunidade, equipe de trabalho, público atendido e relação da comunidade com os serviços de saúde.
As comunidades entrevistadas foram localizadas por meio de cadastro junto ao Fórum Goiano de Enfrentamento ao Crack e Outras Drogas e ao Grupo Executivo de Enfretamento às Drogas - GEED. O trabalho de campo foi realizado por meio da aplicação do questionário na sede da instituição. As visitas às instituições foram realizadas no período compreendido entre os meses de novembro de 2013 a fevereiro de 2015.

$\mathrm{Na}$ entrevista, o responsável pela instituição lia e assinava o Termo de Consentimento Livre e Esclarecido TCLE, que garante o sigilo e a identidade do participante. Esta pesquisa está inscrita na Comissão Nacional de Ética em Pesquisa-CONEP coma CAAE: 12416213.1.0000.0037 e foi aprovada pelo Comitê de Ética em Pesquisa - CEP da Pontifícia Universidade Católica de Goiás - PUC Goiás.

Após a aplicação dos questionários, foi confeccionado um banco de dados utilizando-se do software estatístico Statistical Package for the Social Science - SPSS versão 16. As variáveis referentes ao objeto de estudo do presente artigo foram destacadas e os resultados obtidos por meio de estatística descritiva.

\section{RESULTADOS}

Um total de 43 comunidades terapêuticas CTs foram pesquisadas, sendo 36 (83,7\%) destinadas exclusivamente ao público masculino e 7 (16,3\%) ao público feminino. Nas comunidades masculinas, havia uma média de 26,92 ( $\pm 27,13$ ) residentes, tendo uma quantidade mínima de 3 e a máxima de 150 . Já nas comunidades femininas, havia uma média de 7,57 ( \pm 3,87 ), com a quantidade mínima de 2 e máxima de 12 mulheres residentes no momento da pesquisa. A distribuição dos residentes em cada comunidade segue nas tabelas 1 e 2 abaixo: 
Tabela 1. Quantidade de Residentes em cada Comunidade (masculina)

\begin{tabular}{|c|c|}
\hline Número da Comunidade & Quantidade de residentes \\
\hline 1 & 3 \\
\hline 3 & 18 \\
\hline 4 & 6 \\
\hline 5 & 37 \\
\hline 6 & 48 \\
\hline 7 & 12 \\
\hline 8 & 24 \\
\hline 9 & 80 \\
\hline 10 & 22 \\
\hline 11 & 33 \\
\hline 12 & 80 \\
\hline 15 & 19 \\
\hline 16 & 15 \\
\hline 17 & 16 \\
\hline 18 & 18 \\
\hline 19 & 37 \\
\hline 20 & 24 \\
\hline 21 & 5 \\
\hline 22 & 7 \\
\hline 23 & 22 \\
\hline 25 & 31 \\
\hline 26 & 16 \\
\hline 27 & 23 \\
\hline 28 & 7 \\
\hline 29 & 30 \\
\hline 30 & 22 \\
\hline 31 & 20 \\
\hline 33 & 21 \\
\hline 34 & 20 \\
\hline 35 & 13 \\
\hline 36 & 13 \\
\hline 37 & 23 \\
\hline 39 & 150 \\
\hline 40 & 20 \\
\hline 42 & 29 \\
\hline 43 & 5 \\
\hline Total de residentes & 969 \\
\hline
\end{tabular}

Tabela 2. Quantidade de Residentes em cada Comunidade (feminina)

\begin{tabular}{cc}
\hline Número da Comunidade & Quantidade de residentes \\
\hline 2 & 8 \\
13 & 3 \\
14 & 10 \\
24 & 11 \\
32 & 2 \\
38 & 7 \\
41 & 12 \\
\hline Total de residentes & $\mathbf{5 3}$ \\
\hline
\end{tabular}

Dentre as 43 comunidades pesquisadas, $27,9 \%$ possuíam mantenedora e $72,1 \%$ não tinham. Dentre as CTs pesquisadas $53,5 \%$ afirmaram que tinham uma orientação do trabalho com sentido ligado à religião, 30,2\% somente comunitário e $14 \%$ religioso e comunitário. A maioria do público atendido pelas CTs era de dependentes químicos de drogas lícitas e ilícitas representando $97,7 \%$ das comunidades.

Com relação à sede da comunidade tivemos $51,2 \%$ com sede própria, 30,2\% com sede alugada e $18,6 \%$ com sede cedida, sendo que todas as comunidades visitadas $(100 \%)$ eram entidades não governamentais. Estas, em sua maioria, apresentavam residentes oriundos somente da zona urbana, representado por $95,3 \%$ das CTs, sendo que apenas 2,3\% tinham como residentes apenas da zona rural e 2,3\% da zona rural e urbana. A renda familiar média desses residentes variava entre $1 \mathrm{a}$ 5 salários mínimos, apresentando 53,5\% com ganho de 1 salário mínimo e 46,5\% com ganho entre 2 a 5 salários mínimos.

Com relação ao serviço de atenção à saúde, para o atendimento aos problemas de saúde dos residentes, as 43 CTs (100\%) pesquisadas se utilizavam do Sistema Único de Saúde - SUS, principalmente nos seguintes serviços de saúde: odontológico (86,1\%) e médicos de variadas especialidades com a realização de exames laboratoriais e também com serviços de urgência (86\%).

A utilização dos serviços oferecidos pelo SUS se mostrou bastante frequente pelas CTs, demostrando uma preocupação com a saúde física de seus residentes. Um total de $48,8 \%$ das CTs encaminha semanalmente seus residentes a algum serviço de saúde e mensalmente 
representam 34,9\%. Em todas as CTs (100\%) há o acompanhamento dos residentes nos serviços de saúde por alguém, sendo na sua grande maioria acompanhamentos por pessoas ou funcionários ligados à CT.

Havia comunidades que recebiam residentes encaminhados por outras instituições ou serviços de saúde representando a maioria das CTs visitadas 90,7\%, e apenas 9,3\% nunca receberam esses internos. Quando perguntado sobre a frequência com que elas recebiam residentes, a maioria era semanalmente e mensalmente representada por $56,4 \%$ das CTs, e bimestralmente por $20,5 \%$, semestralmente por $12,8 \%$, anualmente ou menos por $10,3 \%$.

Já em relação ao movimento contrário, quando o residente é encaminhado de sua CT para outras instituições, comunidades ou serviços de saúde, identificou-se que 83,7\% das CTs já haviam encaminhado, enquanto $16,3 \%$ delas nunca encaminharam. A frequência dos encaminhamentos também foi questionada, mostrando que a maioria das CTs encaminhava mensalmente $27,8 \%$, semanalmente $16,7 \%$, totalizando $44,4 \%$ sendo uma boa frequência. Já bimestralmente e semestralmente apresentaram a mesma frequência de $22,2 \%$.

Quando perguntado sobre o recebimento de residentes ou ex-residentes encaminhados diretamente pelo Centro de Atenção Psicossocial - CAPS, 50\% das comunidades responderam que já tinham recebido e $50 \%$ nunca receberam. Muitos desses recebimentos eram motivados por ordem judicial, pelo hospital, mau estado ou transtorno mental, por conta própria do dependente químico e quando era morador de rua.

Já em relação ao movimento contrário, ou seja, encaminhamento de residentes ou ex-residentes das CTs para o CAPS o estudo identificou que $67,4 \%$ encaminhavam ou já encaminharam os residentes enquanto que 32,6\% nunca fizeram o uso desse serviço. Dentre os motivos apontados pelas CTs para encaminhar seu residente ao CAPS destacam-se aqueles serviços próprios do CAPS que é de atenção à saúde mental. Assim, as CTs apontaram como motivos, por exemplo, complicação mental e psicológica (18,2\%), suporte psicossocial e medicação (9\%), consultas psiquiátricas $(40,9 \%)$, dentre outras motivações.

\section{DISCUSSÃO}

Identifica-se nos dados apresentados um perfil muito bem delineado das comunidades terapêuticas e a população atendida por elas. Ou seja, de entidades não governamentais com forte cunho religioso que visam o tratamento de forma voluntária de dependentes de drogas. Observa-se também que tal perfil se direciona ao atendimentoaindivíduoscombaixonívelsocioeconômico, como apresenta a pesquisa de Reis, Uchimura e Oliveira (2013), que demonstrou o baixo nível de renda familiar associado ao uso de drogas em sua pesquisa feita em uma comunidade com elevados indicadores de violência relacionados ao consumo de drogas. Esse é um fator importante no papel desempenhado pelas comunidades no trabalho com os dependentes químicos.

As instituições religiosas possuem um importante papel na ajuda das comunidades. Essas entidades religiosas, na maioria dos casos são mantenedoras das CTs, possuem o objetivo de promover a propagação do Evangelho de Jesus Cristo, com base nos ensinamentos bíblicos. Tal atuação visa à reabilitação física, psíquica, emocional e espiritual e a reinserção social. Na perspectiva das entidades religiosas, os programas oferecidos nessas CTs têm como foco as mudanças comportamentais e a qualidade de vida dos usuários de substâncias químicas e de seus familiares (SILVA, 2013).

A elevada quantidade de dependentes químicos oriundos da zona urbana se relaciona com o fenômeno urbano da proliferação das drogas e dos guetos em que os dependentes se relacionam. Dessa maneira, é de compreender que a maioria das CTs busca a sua sede em lugares mais isolados justamente para o interno se reencontrar com o meio rural, com o plantio de alimento, com a lida de animais domésticos (DAMAS, 2013).

O Sistema Único de Saúde - SUS garante o acesso integral, universal e gratuito para toda a população do país, sendo criado em 1988 pela Constituição Federal brasileira. Dessa forma, é um sistema que trabalha com os princípios da Universalidade, da Equidade e Integralidade da atenção à saúde da população. O primeiro princípio consiste em garantir o acesso à saúde a todos os brasileiros. Já o segundo princípio tem como objetivo de fazer $o$ atendimento prioritário para aquelas pessoas 
mais necessitadas. O último princípio tem como base oferecer o suporte de promoção, prevenção, assistência e recuperação da saúde do indivíduo (TEIXEIRA, 2011). Dessa maneira, as CTs possuem não somente o direito da utilização do SUS para seus residentes, mas também o dever de participar desse sistema de saúde com o objetivo de auxiliar na reabilitação do dependente químico e sua família.

A RDC 29/2011 no seu Art. 8 determina que as instituições devem possuir mecanismos de encaminhamento dos residentes à rede de saúde para aqueles que apresentarem intercorrências clínicas decorrentes ou associadas à privação ou ao uso de substâncias psicoativas, como também para aqueles que apresentarem outros agravos à saúde. Dessa forma, as CTs têm obrigação de levar o residente a um atendimento de saúde, seja de atenção primária ou de urgência, para que o paciente seja avaliado por uma equipe médica. Assim, caso precise de um serviço médico especializado, este é regulado a um hospital.

No entanto, como mostra Silva (2013), o Relatório de Avaliação das Condições Higiênico-Sanitárias das Comunidades Terapêuticas no Estado de Santa Catarina apresentou que apenas $40 \%$ das CTs ofereciam esse tipo de suporte à saúde dos residentes e $77 \%$ utilizavam a Rede SUS para encaminhamento de intercorrências clínicas decorrentes ou associadas ao uso ou privação de substâncias, ou para os outros agravos à saúde.

Sendo assim, observa-se que as CTs pesquisadas na sua totalidade estão fazendo a interlocução com os diversos serviços de saúde, sendo que esses encaminhamentos, na maioria das CTs, eram feitos com elevada frequência, demonstrando uma preocupação com o estado de saúde dos residentes. É importante salientar também que todos os encaminhamentos eram para o SUS e sempre possuíam um acompanhante da comunidade com os residentes durante a ida para a unidade de saúde.

O CAPS tem um papel, dentro da política pública de atendimento à saúde mental, de atenção psicossocial a pacientes com transtornos decorrentes do uso e dependência de substâncias psicoativas servindo, assim, como uma porta de entrada para o usuário à rede de assistência local de atendimento. Tem como ênfase o tratamento na redução de danos e não na internação do usuário (COSTA, 2009).

Além disso, o CAPS também atua na reinserção dos usuários na vida social. Isso feito através do acesso ao trabalho, lazer, exercício dos direitos civis e fortalecimento dos laços familiares e comunitários (BRASIL, 2011c). Tais fatos mostram uma importante articulação que deveria existir entre o CAPS e as CTs, já que o CAPS funcionando como porta de entrada para dependentes químicos encaminharia aqueles que quisessem, voluntariamente, ser internados em CTs para o fim da dependência. Por outro lado, as CTs também encaminhariam ao CAPS aqueles residentes que necessitassem de atendimento psiquiátrico e psicológico mais especializado.

Uma pesquisa de Silva (2013) apresentou que havia ainda muitas brechas entre a articulação entre o CAPS e as CTs. Nele mostrou que muitos usuários não se adequavam ao tratamento ambulatorial presente nos CAPS e por isso não tinham seu tratamento correspondido no enfrentamento à dependência. No entanto, eles também não recebiam acolhimento ou informação para serem encaminhados às CTs para o tratamento voluntário baseado em uma internação. Entretanto aqueles residentes que recebiam a informação das CTs eram transferidos para elas, onde havia melhora do quadro de dependência.

Silva (2011) em uma pesquisa feita em uma CTs da região metropolitana de São Paulo mostrou o benefício da forte articulação entre a comunidade terapêutica e os centros de saúde da região. Estes foram responsáveis pelo encaminhamento de $28 \%$ dos residentes, sendo que a comunidade também possuía um vínculo bastante forte com o município, já que este através do projeto Viva Luz que buscava moradores de rua foram responsáveis por $65 \%$ das internações da comunidade. A prefeitura também auxiliava no mercado de trabalho após a alta dos residentes, já que ela oferecia trabalho para cuidar de lugares públicos, praças, escolas. Isso mostra a importância da articulação das CTs com a área da saúde e também com serviços públicos locais.

No presente estudo, podemos observar claramente que há uma relativa articulação entre as CTs visitadas e o CAPS, já que a maioria delas encaminha residentes para o CAPS. No entanto, o inverso não foi 
observado na maioria das CTs, pois apenas metade das comunidades recebe pacientes encaminhados pelos CAPS.

\section{CONCLUSÃO}

O que se conclui é que as comunidades terapêuticas mesmo se utilizando dos serviços de saúde para o tratamento dos residentes, não faz seu uso de maneira efetiva, ou seja, ainda há muitos recursos do SUS que as comunidades terapêuticas deveriam se utilizar para o tratamento eficiente do dependente químico como, por exemplo, a articulação com os CAPS. Os CAPS possuem importantes ferramentas que podem auxiliar as comunidades como: profissionais capacitados para o atendimento de distúrbios psiquiátricos e psicológicos e também medidas de reinserção do indivíduo à sociedade, através de serviços e programas que auxiliam essa aproximação social do dependente químico.

Atualmente, a relação entre os serviços de saúde e comunidades terapêuticas da cidade de Goiânia (GO), sua região metropolitana e Anápolis (GO) funciona com o acesso a médicos de variadas especialidades, odontólogos, psiquiatras e psicólogos. No entanto, ainda urge a necessidade de estabelecer mais vínculos entre as comunidades e esses serviços, haja vista que não são todas que possuem essa relação consolidada.

Ao que se observa das políticas públicas existentes que envolvem o dependente químico, a efetiva aplicação de suas ações já previstas merece maior atenção por parte dos gestores governamentais, visando uma maior interlocução dos vários serviços oferecidos ao sujeito de direitos e seus familiares que necessitam do atendimento e de informações precisas sobre os equipamentos e atividades proporcionadas. Há uma rede de atendimento que necessita ser aplicada e seus agentes atuarem de forma sistematizada, na interlocução destas ações e na complexidade do atendimento ao dependente químico.

\section{AGRADECIMENTOS}

Agradecemos à Fundação de Amparo à Pesquisa do Estado de Goiás (FAPEG) pelo financiamento da pesquisa, à Pontifícia Universidade Católica de Goiás (PUC Goiás) pelas Bolsas de Iniciação Científica concedidas, e também à Coordenação de Aperfeiçoamento de Pessoal de Nível Superior (CAPES) pela Bolsa de Mestrado concedida.

\section{REFERÊNCIAS}

BRASIL. Portaria $\mathrm{n}^{\mathbf{0}}$ 3088, de 23 de dezembro de 2011. Institui a Rede de Atenção Psicossocial para pessoas com sofrimento ou transtorno mental e com necessidades decorrentes do uso de crack, álcool e outras drogas, no âmbito do Sistema Único de Saúde. Ministério da Saúde, 2011a. Disponível em: <http://www.brasilsus.com.br/ legislacoes/g m/1112763088.html>. Acesso em: 12 mar. 2014.

BRASIL. RDC ANVISA no 29, de 30 de junho de 2011. Dispõe sobre os requisitos de segurança sanitária para o funcionamento de instituições que prestem serviços de atenção a pessoas com transtornos decorrentes do uso, abuso ou dependência de substâncias psicoativas. Ministério da Saúde, 2011b. Disponível em: <http:// bvsms.saude.gov.br/bvs/saudelegis/navisa/2011/ res0029_30_06_2011.htm >. Acesso em: 09 mar. 2014.

BRASIL. Observatório Crack, é possível vencer. Ministério da Saúde, 2011c. Disponível em: < http://www. brasil.gov.br/observatoriocrack/cuidado/outros-centrosatencao-psicossocial.html>. Acesso em: 01 jul. 2015.

COSTA, S. F. As políticas públicas e as comunidades terapêuticas nos atendimentos à dependência química. Disponível em: <http://www.uel .br/revistas/ ssrevista/pdf/2009/29\%20AS\%20POL\%CDCAS\%20 P\%DABLICAS\%20E\%20AS\%20COMUNIDAD\%20 TERAP\%CAUTICASCOM\%20REVIS\%C3O\%20DO\%20 AUTOR.pdf>. Acesso em: 20 mar. 2015.

DAMAS, F. B. Comunidades terapêuticas no Brasil: expansão, institucionalização e relevância social. Rev Saúde Pública, Florianópolis, v. 6, n. 1, p. 50-65, jan/ mar. 2013. 
DE LEON, G. A comunidade terapêutica: teoria, modelo e método. São Paulo: Loyola, 2003.

DIAS, A. C.; ARAÚJO, M. R.; LARANJEIRA, R. Evolução do consumo de crack e coorte com histórico de tratamento. Rev Saúde Pública, São Paulo, v. 45, n. 5, p. 938-948, out. 2011.

FERREIRA, P. S.; LUIS VILLAR, M. A. Percebendo as facilidades e dificuldades na implantação de serviços abertos em álcool e drogas. Texto Contexto - Enferm., v. 13, n. 5, p. 209-216, abr/jun. 2004.

MORSELLI, V.; REZENDE, S.; ROCHA, S. Cadastro Estadual das Comunidades Terapêuticas e Instituições Afins - Goiás. Eixo das Comunidades Terapêuticas. Fórum Goiano de Enfrentamento ao Crack e outras drogas. [S.l.; s.n.], 2012.

RAUPP, L. M.; MILNITISKY-SAPIRO, C. A. "Reeducação" de adolescentes em uma comunidade terapêutica: o tratamento da drogadição em uma instituição religiosa. Psicologia: Teoria e Pesquisa. Brasília, 2008, v. 24, n. 3, p. 361-368.

REIS, L. M.; UCHIMURA, T. T.; OLIVEIRA, M. L. F. Perfil socioeconômico e demográfico em uma comunidade vulnerável ao uso de drogas de abuso. Acta Paul Enferm., v. 26, n. 5 , p. $276-282,2013$.

RIBEIRO, M.; FIGLIE, N. B.; LARANJEIRA, R. Organização de serviços de tratamento para a dependência química. In: FIGLIE, N. B.; BORDIN, S.; LARANJEIRA, R. (Org.). Aconselhamento em dependência química. São Paulo, 2004.

SILVA, M. A. R. Comunidade terapêutica: na mão ou na contramão das reformas sanitária e psiquiátrica? 2013. 265f. Dissertação (Mestrado Profissional em Saúde Mental e Atenção Psicossocial) - Universidade Federal de Santa Catarina Centro de Ciências da Saúde, Florianópolis, 2013.

SILVA, J. L. Terapia de rede para adictos: Programa de tratamento e prevenção para dependentes de drogas em comunidades terapêuticas. 2011. 225f. Tese (Programa de Pós-Graduação em Saúde Pública) - Universidade de São Paulo, Faculdade de Saúde Pública, São Paulo, 2011.
TEIXEIRA, C. Os princípios do Sistema Único de Saúde 2011. Disponível em: <http://www.saude.ba.gov. br/pdf/OS_PRINCIPIOS_DO_SUS.pdf $>$. Acesso em: 03 jun. 2015.

UNODC. Relatório mundial sobre drogas de 2012. United Nations Office on drugs and crime. Disponível em: $<$ http://www.uno dc.org/southerncone/pt/drogas/relatori o-mundial-sobre-drogas.html> Acesso em: 03 mar. 2014.

UNODC. Relatório mundial sobre drogas de 2013. United Nations Office on drugs and crime. Disponível em: <http://www. unodc.org/southerncone/pt/ drogas/ relatorio-mundial-sobre-drogas.html> Acesso em: $25 \mathrm{fev}$. 2014.

Recebido em: 18 de agosto de 2015 Aceito em: 13 de setembro de 2015 